\title{
Pedunculated mucinous cystic neoplasm of the liver: a case report
}

\author{
Sang-Woo Ha ${ }^{1}$, Shin Hwang ${ }^{1}$, Hyejin $\mathrm{Han}^{1}$, Song Ie Han ${ }^{1}$, Seung-Mo Hong ${ }^{2}$ \\ ${ }^{1}$ Department of Surgery, Asan Medical Center, University of Ulsan College of Medicine, Seoul, Korea \\ ${ }^{2}$ Department of Pathology, Asan Medical Center, University of Ulsan College of Medicine, Seoul, Korea
}

In 2010, the World Health Organization classified mucin-producing bile duct tumors of the liver into two distinct entities; mucinous cystic neoplasm of the liver (MCN-L) and intraductal papillary mucinous neoplasm of the bile duct. We present the case of a patient with MCN-L having a uniquely pedunculated shape. A 32-year-old woman was referred to our institution with a diagnosis of biliary cystic neoplasm. She had undergone left salpingo-oophorectomy for ovarian cancer 15 years ago. Imaging studies showed an $8 \mathrm{~cm}-$ sized well defined, multiloculated cystic lesion suggesting a mucinous cystic neoplasm. The cystic mass was pedunculated at the liver capsule and pathologically diagnosed as MCN-L. The mass was resected with partial hepatectomy. The patient recovered uneventfully. She was discharged 7 days postoperatively. The patient has been doing well for 6 months after the operation. The patient will be followed up annually because of the favorable postresection prognosis of MCN-L.

Keywords: Cystic neoplasm; Intraductal papillary neoplasm; Mucins; Ovarian-like stroma; WHO classification

\section{Introduction}

In 2010, the World Health Organization (WHO) classified mucin-producing bile duct tumors of the liver into two distinct entities: mucinous cystic neoplasm of the liver (MCN-L) and intraductal papillary mucinous neoplasm of the bile duct (IPMN-B) $[1,2]$. MCN-L is a rare disease that accounts for less than $5 \%$ of all cystic liver lesions [2-6]. This disease predominantly occurs in women $[1,4,7]$. Histologically, it is defined as a cyst-forming epithelial neoplasm composed of mucin-producing epithelium associated with ovarian-like stroma (OLS) [2]. MCN-L is graded as low-, intermediate-, and high-grade intraepithelial dysplasia, carcinoma in situ, or associated invasive carcinoma $[1,2,8]$.

MCN-L has previously been known as biliary cystadenoma or cystadenocarcinoma [3]. We have previously reported 23 cas- es of biliary cystadenoma and seven cases of biliary cystadenocarcinoma [9], for which the definition of MCN-L according to the WHO 2010 classification was not adopted because these study patients had undergone surgical resection between 2007 and 2013.

We present the case of a patient who underwent hepatic resection for $\mathrm{MCN}-\mathrm{L}$ with a uniquely pedunculated shape.

\section{Case}

Ethical statements: This study was approved by the Institutional Review Board (IRB) of Asan Medical Center (IRB No: 2021-0839), and informed consent was obtained from the patient.

Received: June 17, 2021 • Revised: June 25, 2021 • Accepted: July 3, 2021

Corresponding author: Shin Hwang, MD, PhD, FACS

Department of Surgery, Asan Medical Center, University of Ulsan College of Medicine, Olympic-ro 43-gil 88, Songpa-gu, Seoul 05505, Korea

Tel: +82-2-3010-3930• Fax: +82-2-3010-6701・E-mail: shwang@amc.seoul.kr 

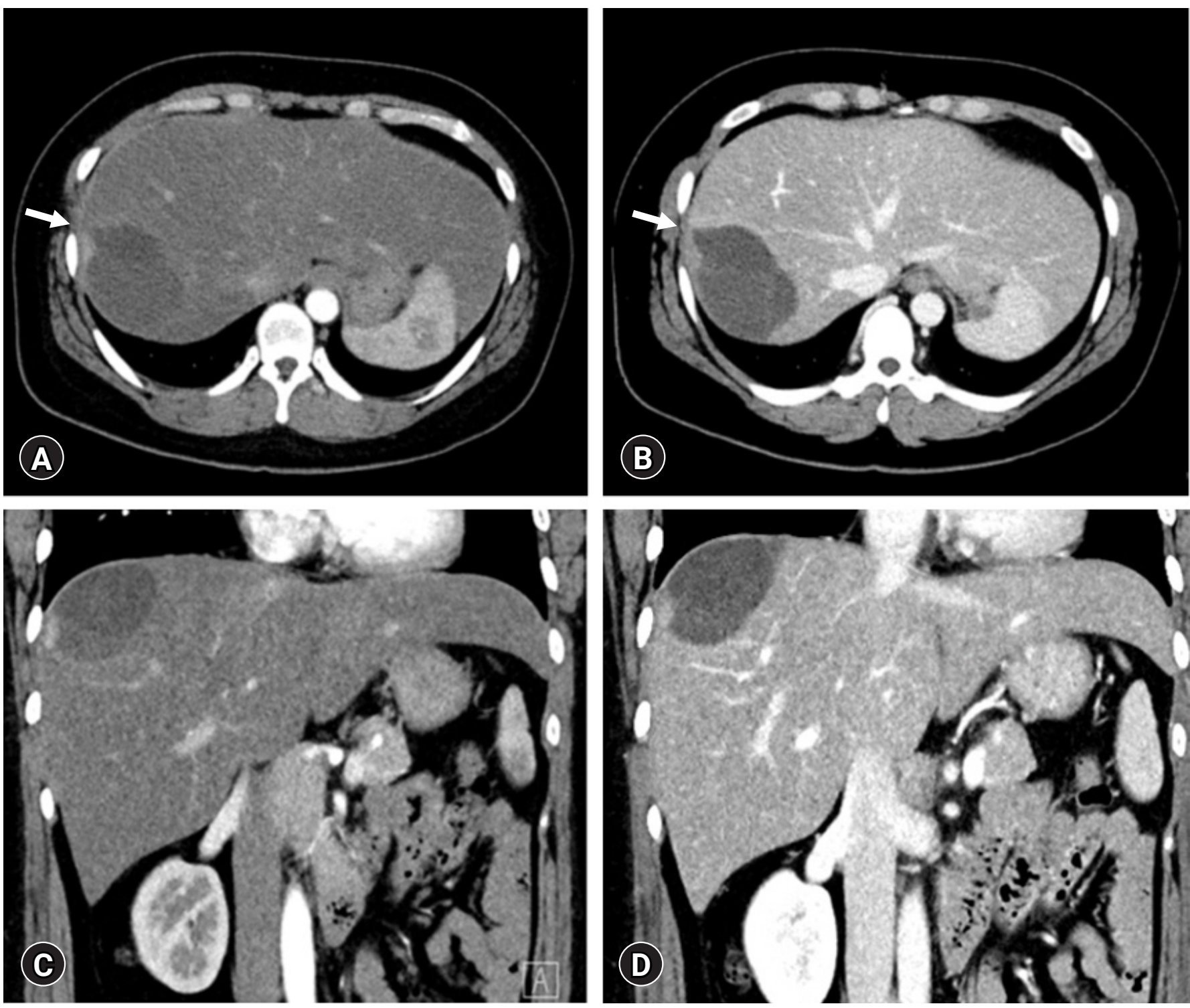

Fig. 1. Preoperative computed tomography showing an $8 \mathrm{~cm}$-sized well defined, multiloculated cystic lesion. There is some high attenuation portion within the cystic lesion indicating hemorrhage (arrows) in $(A, C)$ the arterial phase and $(B, D)$ portal phase images. Diffuse fatty liver is present.

A 32-year-old woman was referred to our institution with a diagnosis of biliary cystic neoplasm. The patient underwent computed tomography (CT) at a local hospital because of a sudden onset of upper abdominal pain persisting for 1 day. The abdominal CT showed an $8 \mathrm{~cm}$-sized multiseptated cystic mass with some high attenuation fluid in the right lobe of the liver, suggesting a biliary cystic neoplasm. She had undergone left salpingo-oophorectomy for ovarian cancer 15 years ago.

A repeat abdominal CT scan showed an $8 \mathrm{~cm}$-sized well defined, multiloculated cystic lesion, suggesting a mucinous cystic neoplasm (Fig. 1). A high attenuation portion was observed within the cystic lesion, indicating hemorrhage rather than a thickened septum. There was no attenuation change in the surrounding liver tissue or bile duct dilatation. Diffuse fatty liver and splenomegaly were present. Fluorodeoxyglucose positron emission tomography-CT showed an $8 \mathrm{~cm}$-sized metabolic defect in the right liver (Fig. 2). In the right ovary, a cystic lesion with mild hypermetabolic uptake was observed. Pelvic ultrasonography performed at the Department of Obstetrics and Gynecology showed a $3.5 \mathrm{~cm}$-sized cystic lesion in the right adnexa, which was an indication for further follow-up. There was no evidence of ovarian cancer recurrence or liver metastasis.

Because the patient had undergone surgery for ovarian cancer, she wanted to receive an upfront surgery for liver mass resection. 


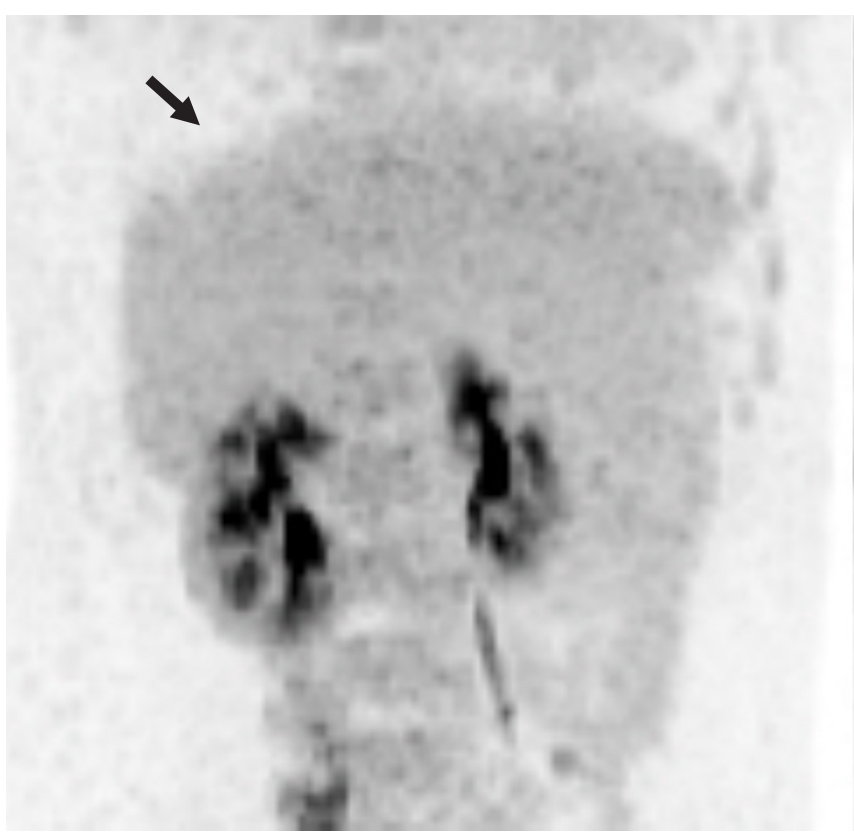

Fig. 2. Fluorodeoxyglucose positron emission tomography showing an $8 \mathrm{~cm}$-sized metabolic defect in the right liver (arrow).

We planned to perform a partial hepatectomy to remove the cystic mass. After laparotomy, the gallbladder was resected because of previous surgery-associated adhesions. There were noticeable adhesions around the cystic mass in the right dome of the liver. After meticulous dissection of the liver dome, we unexpectedly identified that the cystic mass was uniquely pedunculated in the liver capsule (Fig. 3). The cystic mass compressed the liver parenchyma; thus, the corresponding portion was depressed to hold it. We presumed that hemorrhagic expansion of the cystic mass was associated with the sudden onset of upper abdominal pain. The mass was delivered after partial hepatectomy in the pedunculated neck portion. The right ovarian cyst was assessed intraoperatively by obstetricians and regarded as a functional ovarian cyst.

The pathology report revealed that the cystic lesion was multiloculated and filled with mucinous fluid (Fig. 4). The diagnosis was a low-grade MCN-L of $8 \times 6 \times 4 \mathrm{~cm}$ that was attached to the Glisson capsule. There was no finding of a usual discolored hematoma in the lumen of the cystic lesion. Focal luminal thickening, which was well matched with the preoperative CT findings, appeared to be suggestive of a resolved hematoma. The cyst was lined by tall columnar tumor cells possessing supranuclear cytoplasmic mucin with elongated nuclei and mild cytologic atypia. Subepithelial OLS cells with elongated nuclei were diffusely positive for estrogen receptors (Fig. 5). Focal positivity was also observed in the OLS cells.

The patient recovered uneventfully (Fig. 6). She was discharged
7 days postoperatively. The patient has been doing well for 6 months after the operation. The patient will be followed up every 6 to 12 months because of MCN-L and ovarian cyst .

\section{Discussion}

MCN-L shows multilocular cysts with septation. It is often located in the left liver. Considering these usual findings of MCN-L, our patient showed a unique feature of pedunculated MCN-L. The cystic mass occupied the space at the liver dome, leaving a counterpart cast-like depression in the liver parenchyma. Such intraoperative findings suggested that the cystic tumor had grown very slowly over a long period. We presumed that hemorrhagic expansion of the cystic mass was associated with preoperative upper abdominal pain, which occurred suddenly and persisted for a day.

MCN-L should be differentiated from IPMN-B, which is recognized as a biliary counterpart to the papillary intraductal mucinous neoplasm of the pancreas. The characteristics of IPMN-B include multicystic appearance, bile duct dilation, the presence of intraluminal masses, and intraductal nodules $[1,5,7,10,11]$. IPNM-B has the typical characteristics of an absence of OLS. It is histologically described as a mucinous and papillary neoplasm, with a clear origin in the biliary epithelium and solitary or diffuse intraductal growth [10]. However, there are some case reports of MCN-L showing the presence of a prolapsed tumor mass in the left hepatic duct and common bile duct that can cause ductal dilation $[10,12]$. These findings suggest that ductal dilation and communication with the bile duct might not be typical signs of IPMN-B because they can be identified in MCN-L.

It is difficult to differentiate between MCN-L and IPMN-B preoperatively. MCN-L usually shows the absence of communication with the bile duct, absence of bile duct dilation, and multilocular shape, but these findings might not always be decisive when defining the diagnosis of MCN-L. The presence of OLS in the histopathological section was established by the WHO 2010 classification as a diagnostic criterion for MCN-L. In contrast, immunohistochemical profiling of mucin core proteins (MUC1, MUC2, and MUC5AC) and cytokeratin (CK7 and CK20) is used to classify IPMN-B into four types; pancreaticobiliary, intestinal, gastric, and oncocytic types $[13,14]$.

There are some common characteristics between MCN-L and IPMN-B, such as mucin production rare incidence. However, some clinicopathological features are more typical of each neoplasm. MCN-L predominantly occurs in females but not IPMN-B $[1,7,12,15]$. It is also difficult to differentiate MCN-L from simple liver cysts. 

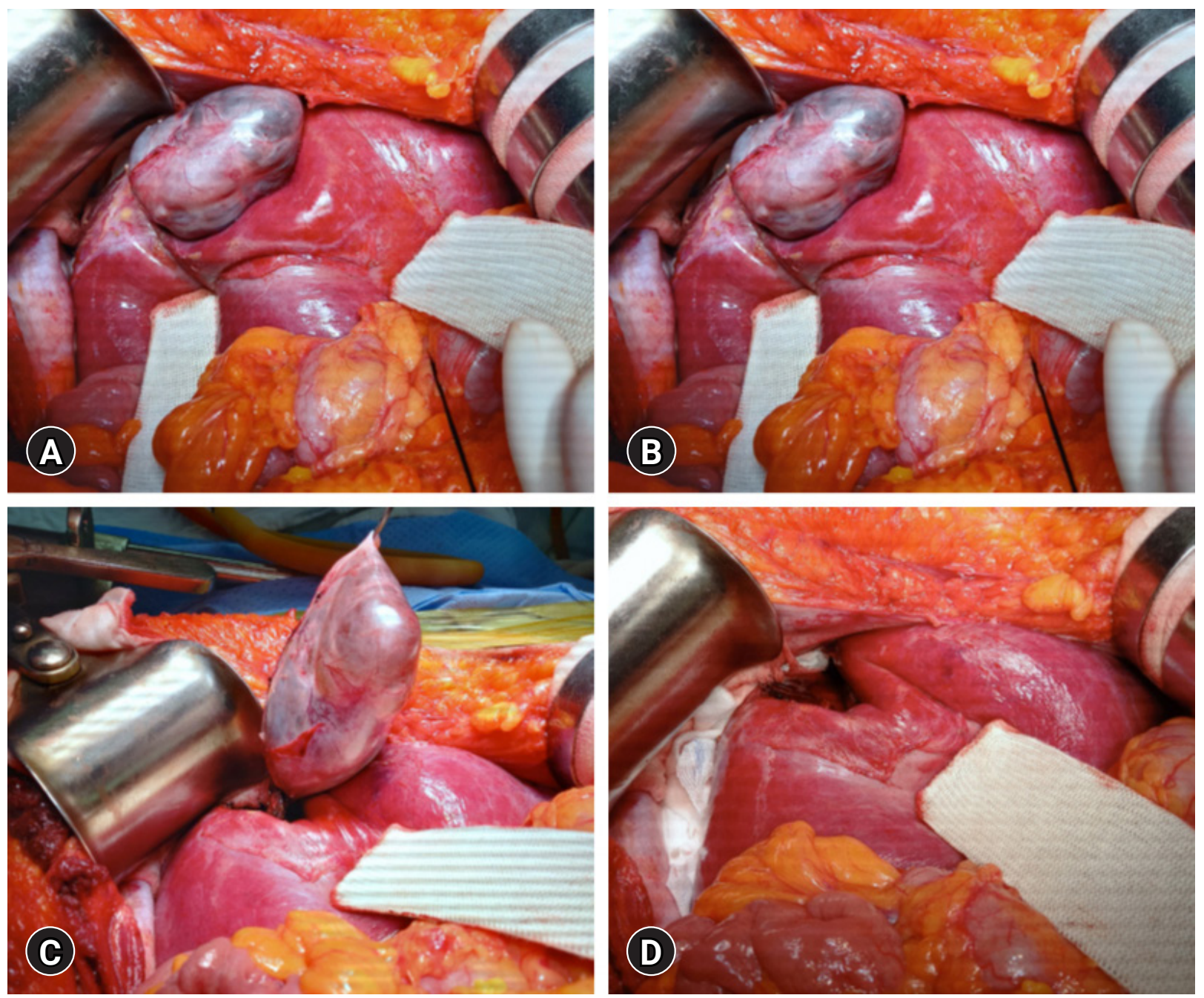

Fig. 3. Intraoperative findings. (A) There are noticeable adhesions around the cystic mass in the right liver dome. (B) The cystic mass is pedunculated at the liver capsule. (C) The mass is delivered after partial hepatectomy at the pedunculated neck portion. (D) A cast-like depression is left at the liver parenchyma.
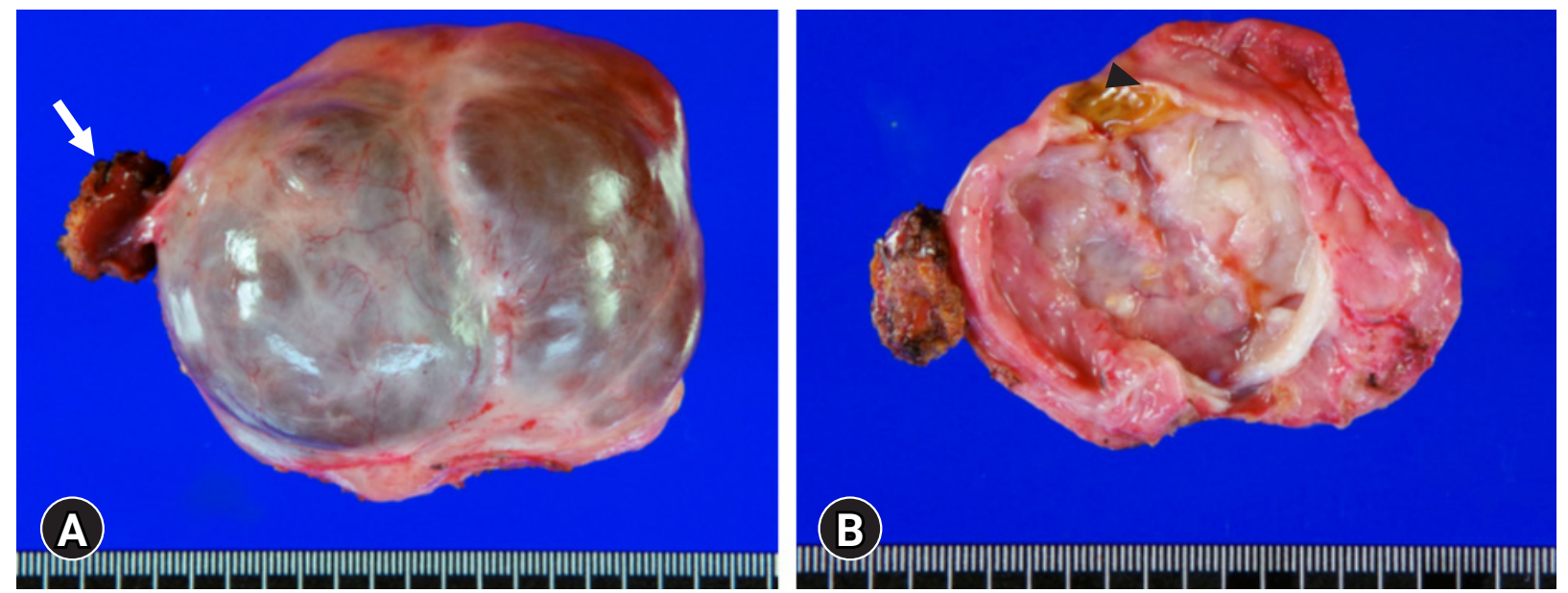

Fig. 4. Gross photographs of the resected specimen. (A) External shape and (B) internal morphology of the cystic mass are visible. The cystic lesion of $8 \times 6 \times 4 \mathrm{~cm}$ is multiloculated and filled with mucinous fluid. Arrow indicates the resected liver parenchyma attached to the neck portion. Arrowhead indicates the focal luminal thickening suggestive of resolved hematoma. 

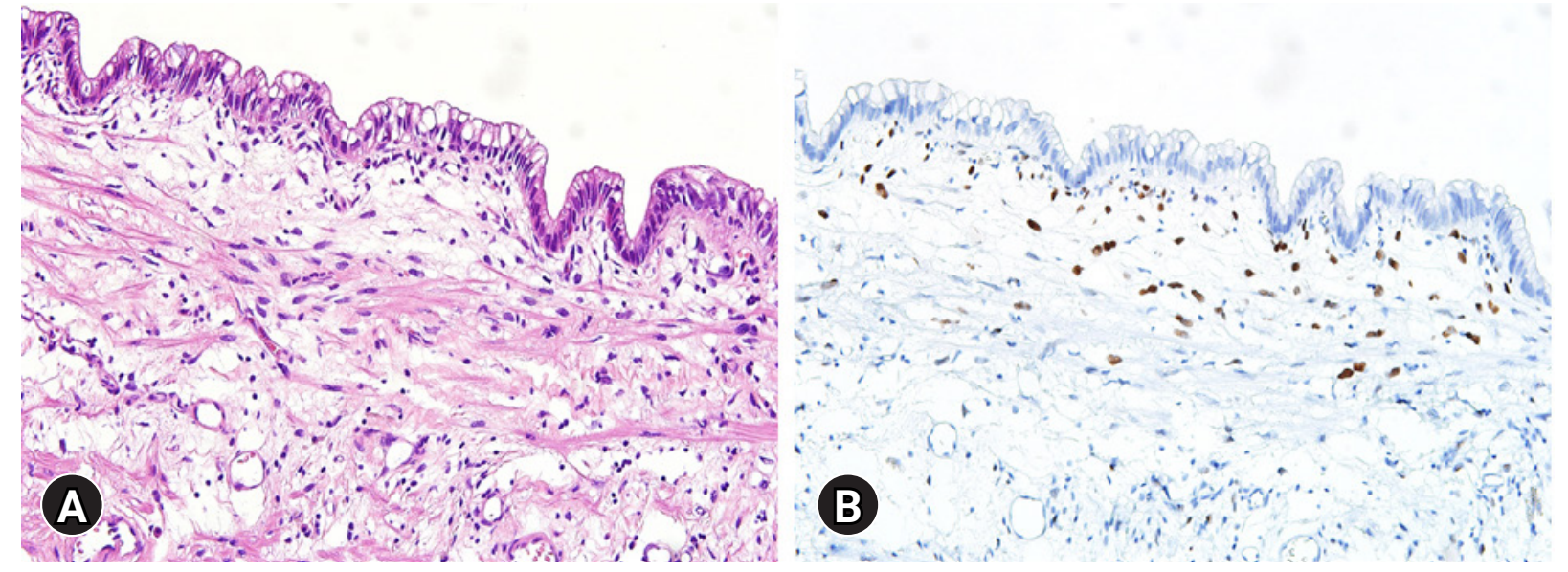

Fig. 5. Microscopic findings. (A) The cyst is lined by tall columnar tumor cells having intracytoplasmic mucin and elongated nuclei with mild cytologic atypia (hematoxylin and eosin stain, $\times 200$ ). (B) The subepithelial ovarian-type stromal cells are diffusely positive for estrogen receptor (immunohistochemical stain, $\times 200$ ).
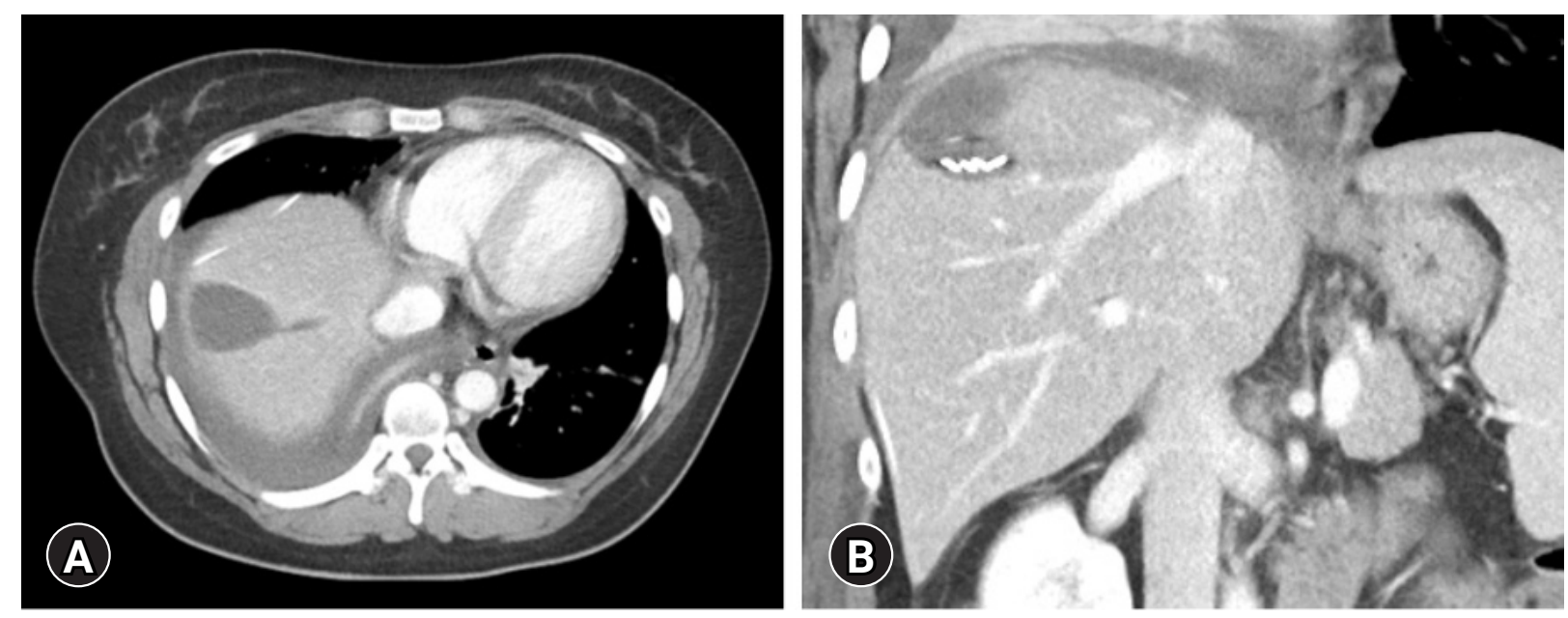

Fig. 6. Computed tomography at 5 days after the operation showing usual postoperative findings. (A) Fluid collection is identified at the site of mass. (B) Multiple metal clips indicate the site of pedunculated neck portion.

A large cyst at the initial presentation, an increase in size during follow-up, and the manifestation of symptoms are general indications for surgical resection $[3,11]$. A 5-year survival rate of $100 \%$ has been reported in patients with MCN-L [1,4]. Considering such favorable postresection outcomes, follow-up protocols for the patient in the present case include the first imaging study at 6 months after resection and subsequent follow-up studies every 12 months for more than 5 years.

\section{Notes}

\section{Conflicts of interest}

No potential conflict of interest relevant to this article was reported.

\section{Funding}

None.

\section{Author contributions}

Conceptualization: SH; Data curation: $\mathrm{SWH}$; Methodology: $\mathrm{HH}$, $\mathrm{SIH}$; Visualization: $\mathrm{SH}, \mathrm{SMH}$; Writing-original draft: $\mathrm{SH}, \mathrm{SWH}$; Writing-review \& editing: all authors.

\section{ORCID}

Sang-Woo Ha, https://orcid.org/0000-0003-0205-5112 Shin Hwang, https://orcid.org/0000-0002-9045-2531 Hyejin Han, https://orcid.org/0000-0002-6091-1717 Song Ie Han, https://orcid.org/0000-0002-2243-3996 Seung-Mo Hong, https://orcid.org/0000-0002-8888-6007 


\section{References}

1. Kubota K, Nakanuma Y, Kondo F, Hachiya H, Miyazaki M, Nagino M, et al. Clinicopathological features and prognosis of mucin-producing bile duct tumor and mucinous cystic tumor of the liver: a multi-institutional study by the Japan Biliary Association. J Hepatobiliary Pancreat Sci 2014;21:176-85.

2. Obara S, Nomi T, Yamato I, Hokuto D, Yasuda S, Nishiwada S, et al. Laparoscopic resection of a hepatic mucinous cystic neoplasm: a case report. Int J Surg Case Rep 2016;24:18-21.

3. Nakayama Y, Kato Y, Okubo S, Takahashi D, Okada R, Nishida $\mathrm{Y}$, et al. A case of mucinous cystic neoplasm of the liver: a case report. Surg Case Rep 2015;1:9.

4. Li T, Ji Y, Zhi XT, Wang L, Yang XR, Shi GM, et al. A comparison of hepatic mucinous cystic neoplasms with biliary intraductal papillary neoplasms. Clin Gastroenterol Hepatol 2009; 7:586-93.

5. Budzynska A, Hartleb M, Nowakowska-Dulawa E, Krol R, Remiszewski P, Mazurkiewicz M. Simultaneous liver mucinous cystic and intraductal papillary mucinous neoplasms of the bile duct: a case report. World J Gastroenterol 2014;20:4102-5.

6. Ghatak S, Ray S, Sonar PK, Das S, Basu K, Mridha AR, et al. Giant mucinous cystic neoplasms of pancreas and liver with unusual adipose tissue component: a case report. J Gastrointest Oncol 2012;3:353-7.

7. Zen Y, Pedica F, Patcha VR, Capelli P, Zamboni G, Casaril A, et al. Mucinous cystic neoplasms of the liver: a clinicopathological study and comparison with intraductal papillary neoplasms of the bile duct. Mod Pathol 2011;24:1079-89.

8. Simo KA, Mckillop IH, Ahrens WA, Martinie JB, Iannitti DA,
Sindram D. Invasive biliary mucinous cystic neoplasm: a review. HPB (Oxford) 2012;14:725-40.

9. Jwa EK, Hwang S. Clinicopathological features and post-resection outcomes of biliary cystadenoma and cystadenocarcinoma of the liver. Ann Hepatobiliary Pancreat Surg 2017;21:107-13.

10. Wang X, Cai YQ Chen YH, Liu XB. Biliary tract intraductal papillary mucinous neoplasm: report of 19 cases. World J Gastroenterol 2015;21:4261-7.

11. Zen Y, Jang KT, Ahn S, Kim DH, Choi DW, Choi SH, et al. Intraductal papillary neoplasms and mucinous cystic neoplasms of the hepatobiliary system: demographic differences between Asian and Western populations, and comparison with pancreatic counterparts. Histopathology 2014;65:164-73.

12. Takano Y, Nagahama M, Yamamura E, Maruoka N, Mizukami $\mathrm{H}$, Tanaka J, et al. Prolapse into the bile duct and expansive growth is characteristic behavior of mucinous cystic neoplasm of the liver: report of two cases and review of the literature. Clin J Gastroenterol 2015;8:148-55.

13. Kim KM, Lee JK, Shin JU, Lee KH, Lee KT, Sung JY, et al. Clinicopathologic features of intraductal papillary neoplasm of the bile duct according to histologic subtype. Am J Gastroenterol 2012;107:118-25.

14. Wan XS, Xu YY, Qian JY, Yang XB, Wang AQ, He L, et al. Intraductal papillary neoplasm of the bile duct. World J Gastroenterol 2013;19:8595-604.

15. Kunovsky L, Kala Z, Svaton R, Moravcik P, Mazanec J, Husty J, et al. Mucinous cystic neoplasm of the liver or intraductal papillary mucinous neoplasm of the bile duct? A case report and a review of literature. Ann Hepatol 2018; 17:519-24. 